Review

\title{
Development challenges for carcinogenicity risk assessments of topical drugs
}

\author{
Brett D Hollingshead'1, Nasir Khan², Maik Schuler² and Zaher Radi1 \\ ${ }^{1}$ Pfizer Drug Safety Research and Development, 1 Portland Street Cambridge, MA, USA \\ ${ }^{2}$ Pfizer Drug Safety Research and Development, 445 Eastern Point Road, Groton, CT, USA
}

[Recommended by Ikuo Horii]

(Received October 25, 2021; Accepted October 25, 2021)

\begin{abstract}
The nonclinical safety package to support development and approval of drugs intended to be administered by topical application generally follows International Council for Harmonisation multidisciplinary 3 (ICH M3) and topic specific safety (ICH S) guidances. However, some aspects of topical drug development may require case-by-case determination of nonclinical safety strategies. The necessity to conduct a dermal rodent carcinogenicity study is one such example that is not considered an obligate component of a nonclinical safety data package for drug approval. While absence of systemic exposure, as stated in ICH M3, is a primary reason to forego a dermal carcinogenicity assessment, there may also be other factors for consideration in determining the need for a life-time carcinogencity study by dermal route to aid in the overall human cancer risk assessment. We therefore reviewed nonclinical carcinogencity data packages from drugs approved by the FDA or PMDA over a $\sim 25$ year time period to evaluate outcomes of oral versus topical carcinogencity studies and to understand their utility for informing the overall human risk assessment. We also discuss various other properties of topical small molecules that could impact the decisions to conduct a dermal life-time rodent carcinogenicity study. Collectively, the need to conduct 2-year dermal carcinogenicity studies in rodents should be determined case-by-case and consider scientific factors such existing systemic toxicity and carcinogenicity study data, anticipated drug exposures in skin, skin evaluation from the chronic minipig toxicity study, and genetic toxicity profile.
\end{abstract}

Key words: Topical carcinogenicity, Aneugen, Skin concentration, Topical drug

\section{INTRODUCTION}

Multiple factors guide a strategy to design and develop medicines intended to be administered by the topical route. Typically, a primary motivation is that the clinical condition or disease intended to be treated manifests in skin and applying a drug directly to the site of interest has the highest likelihood of achieving clinical benefit. It is not uncommon for topical drugs to be reformulations of a compound previously approved or also in clinical development as a systemically bioavailable (e.g., oral or parenteral administration) drug, and the development of novel or reformulated topical drugs may be intended to reduce systemic safety liabilities, maximize concentrations of drugs in the skin, or to increase patient use and compliance (Feldman et al., 2007; Florek et al., 2018; Tieppo
Francio et al., 2017).

The nonclinical safety data packages required to support clinical development and marketing authorization of topical small molecule drugs are generally like those anticipated to be administered by oral and/or parenteral routes. General toxicology, safety pharmacology, genetic toxicity, developmental and reproductive toxicity, and phototoxicity assessments, for example, are usually required to support clinical testing of topical drugs and follow principles outlined in ICH multidisciplinary and discipline specific safety guidance documents. There can also be a need to conduct other specialized studies to characterize skin-related risks for a topical drug. These types of studies, for example, could include in vitro assessments to characterize potential risks related to skin sensitization (OECD, 2018a, 2018b, 2020) or ocular irri-

Correspondence: Brett D Hollingshead (E-mail: Brett.d.hollingshead@pfizer.com) 
B.D. Hollingshead et al.

tancy (OECD, 2009).

One of the final nonclinical studies conducted to support a marketing authorization application for a small molecule is a rodent 2 -year carcinogenicity study. This 'life-time' study in a rodent is the pivotal nonclinical study for most new drug applications used to describe the potential for a drug to have cancer-inducing potential in humans. However, the necessity to conduct a 2 -year carcinogenicity study is not always a straightforward question to answer and a critical evaluation of the need to conduct such studies as a requisite component of overall nonclinical safety packages should be considered on a case-by-case basis (Sistare et al., 2011). An ICH S1B Expert Working Group effort is ongoing to understand when 2-year rodent carcinogenicity studies provide meaningful additional information to support the overall human carcinogenicity risk assessment for systemically bioavailable drugs, compared to when such a study would provide limited additional value to the overall assessment.

It is established that agents possessing certain characteristics, such as endocrine disruption, immune suppression, chronic toxicity, and genotoxic activity, are more likely to possess cancer promoting properties (Monro, 1993). Compounds that contain one or more of these properties often have assumed human risks that would not be further informed by rodent carcinogenicity study results excluding questions related to possible species relevance. Additionally, topically applied drugs without characterized neoplastic risks and/or limited systemic exposure may also have questions around the value in completing rodent carcinogenicity studies.

In this brief review, we have evaluated the pharmacology summary packages and/or labels from 23 topical drug approvals by the United States Food and Drug Administration (FDA) spanning from 1997 through 2020. We additionally have reviewed two recent nonclinical approval packages from topical drugs by the Japan Pharmaceuticals and Medical Devices Agency (PMDA). Collectively, the nonclinical packages for the approved topical drugs that were reviewed highlight that nonclinical carcinogenicity data packages can vary significantly and that conduct of a topical carcinogenicity study should not be viewed as an obligate study required for the approval of all small molecule topical drugs. We further discuss considerations that may be factored on a case-bycase basis to evaluate the necessity to conduct a rodent carcinogenicity study to support topical drug authorization. Proactively identifying scenarios for topical drug development that would not require the need to conduct 2-year studies can help to reduce overall animal usage in drug development programs while maintaining high qual- ity human safety assessments.

\section{CONSIDERATIONS FOR DETERMINING NEED FOR TOPICAL CARCINOGENICITY STUDY}

Physiologic similarity or differences of skin between animals and humans, drug exposures in the viable and/or proliferating compartment of skin, and genotoxicity profile are important factors in considering the needs for a topical carcinogenicity study.

\section{Nonclinical species skin relevance}

Animal-to-human translational relevance is well accepted to be a challenge for understanding human risks derived from animal studies and safety factor adjustments for sensitivity differences between a test species and humans may be incorporated into safety assessments when human translation is uncertain (Dorne and Renwick, 2005). While the rodent (rat, mouse) is a key test system for nonclinical testing of systemically bioavailable drug candidates prior to and during human clinical development, its skin is well known to have significant morphologic and physiologic differences compared to humans (Bartek et al., 1972; Wei et al., 2017).

Unlike the rodent, the minipig has comparatively similar skin morphology and physiology compared to human and is used as the species of choice for dermal evaluation by topical administration in nonclinical toxicity studies (Mortensen et al., 1998; Stricker-Krongrad et al., 2017). Skin in human and minipig is comprised of a relatively thick epidermis and dermis compared to the rodents (Wei et al., 2017). Rodents by contrast have a very dense hair coat with up to $\sim 300-650$ hair follicles per $\mathrm{cm}^{2}$ while the hair follicle density in human and minipig is $\sim 11$ per $\mathrm{cm}^{2}$ (Stricker-Krongrad et al., 2017). The primary barrier protection for skin absorption in humans and minipigs is mediated via the outermost layer of the epidermis, the stratum corneum (SC), which is made up of multiple layers of tightly compacted keratin and horny scales derived from dead keratinocytes (Mortensen et al., 1998). Like the overall epidermis and dermis, the SC in rodents is also thinner than humans or minipigs (Mortensen et al., 1998; Wei et al., 2017). Complete epidermal turnover occurs in normal human and minipig skin is approximately every 4-8 weeks, while the turnover rate in mice is about 1-week (Koster, 2009; Stricker-Krongrad et al., 2017). Given the high density of hair follicles in rodents and thin epidermis it is reasonable to conclude that in rodents the fur coat provides significant barrier protection, and not surprisingly rodent skin itself is generally more permeable to topically applied drugs than human 
Topical carcinogenicity challenges

and minipig (Bartek et al., 1972; Fujii et al., 1997). This is particularly noteworthy when considering that topical rodent studies are conducted by removal of the hair coat so that a test article can be applied directly to the skin. Therefore, it is not surprising that systemic bioavailability by topical application is often higher in rodents compared to human or minipig and can limit the utility and interpretability of rodent models for topical safety evaluations. This is particularly important for topical carcinogenicity studies as skin findings could be difficult to interpret regarding human relevance given the numerous physiological and anatomical species differences.

Collectively, the multifactorial differences between rodent and human skin emphasizes the value of the minipig test system for characterizing safety liabilities of a topical drug candidate in the skin. As discussed in the ICH S1A guideline, a potential cause for concern for carcinogenicity risk (e.g., preneoplastic lesions) can be assessed from routine repeat-dose chronic dermal toxicity studies. Since the epidermis in the minipig turns over approximately once every month (Stricker-Krongrad et al., 2017) a chronic repeat-dose dermal toxicity study of 9-months in duration allows for at least 8 epidermis remodeling events. A careful review of the microscopic evaluation of skin from these minipig studies should be incorporated into the overall carcinogenicity risk assessment. A lack of preneoplastic findings in minipig skin following chronic topical administration may provide supportive information that would mitigate the need to further characterize a topical drug in a 2 -year rodent dermal carcinogenicity study.

\section{Drug concentrations in skin}

A considerable challenge of positioning nonclinical safety skin findings for human risk deals with the prediction of drug concentrations within the skin resulting from clinical use. Systemic toxicity margins are usually derived by measuring plasma drug concentrations as a surrogate for exposure in other tissues (Lehr, 2011). Measurement of plasma drug concentrations in animals and humans with validated bioanalytical methods allows for precise understanding of anticipated concentrations of a compound that would carry a safety concern compared to those achieved when a drug is administered at clinically relevant doses. This information is an important contributor for determining starting doses in first in human clinical trials and to contextualize risks throughout development (Shen et al., 2019).

For topically administered drugs, however, there are currently no standard validated approaches for refined measurements in specific skin compartments. Due to this, safety margins for topical studies are usually not calculated based on exposure but rather are calculated on a dose per unit area (e.g., $\mathrm{mg} / \mathrm{cm}^{2} /$ dose). This reliance on dose margin based calculations underscores the importance of using a test system that is comparatively similar to human, like the minipig, for dermal safety assessments.

Multiple approaches have been described to measure drug concentrations in the skin. A common method is based on homogenization of skin biopsies. While this method can be conducted using standard experimental and analytical techniques it is usually confounded by artifactually high measured drug concentrations introduced via sample collection (Eirefelt et al., 2020; Handler et al., 2021; Surber et al., 1993; Trottet L., 2017). Additionally, a full biopsy homogenate will principally only represent the drug located in the SC or superficial epidermal layers which are nonviable or non-proliferative and rapidly shed, respectively, and therefore are of limited value for safety assessments for threshold mediated mechanisms of toxicity (Hernández et al., 2013; Olaharski et al., 2009). More refined methods for addressing drug concentrations in dermal and or epidermal compartments include microdialysis, open flow microperfusion (OFM), mass spectroscopy imaging, and laser capture microdissection (LCM) approaches (Bodenlenz et al., 2017; Bonnel et al., 2018; Herkenne et al., 2008; Hollingshead et al., 2021).

Given the high amount of active drug that is applied to the skin by topical application, a nominal skin concentration assuming $100 \%$ absorption would be extremely high throughout the skin (e.g., > 1 Molar). This phenomenon, however, is not observed in clinical practice for pharmaceutical drugs since their physiochemical properties are not highly favorable for high skin absorption and steep concentration gradients are observed across the skin. Human and/or minipig concentrations of various drugs in the dermis or epidermis assessed by methods such as OFM, mass spectroscopy imaging, and LCM have illustrated that drug concentrations in viable skin are a fraction of that found in the SC. In humans, for example, studies with OFM have measured average dermal concentrations of an antiviral (acyclovir) and phosphodiesterase inhibitor (LEO 29102) ranging from $\sim 10$ to $1000 \mathrm{nM}$ when applied topically (Bodenlenz et al., 2020; Eirefelt et al., 2020). Likewise, in a pig OFM study, dermal interstitial fluid concentrations of the Janus kinase inhibitors tofacitinib and LEO 27319A were measured at $\leq 100 \mathrm{nM}$ (Handler et al., 2021). A study in minipigs using LCMbased analytical approaches with a topically applied kinase inhibitor demonstrated a very steep concentration gradient within the epidermis, with $>50$-fold lower concentrations in proliferative epidermal stem cells (i.e., stra- 
B.D. Hollingshead et al.

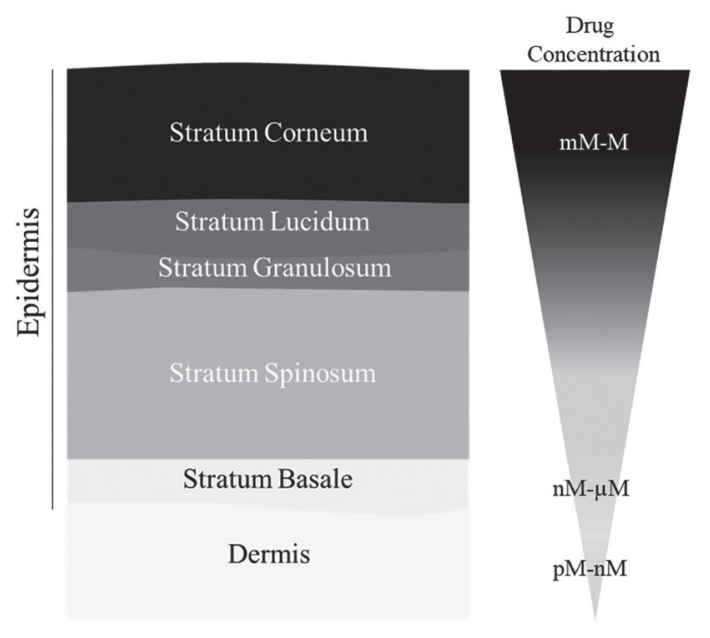

Fig. 1. Drug concentrations in the epidermis and dermis of topical drugs have been shown to follow a steep concentration gradient from the site of application through the epidermal and dermal layers of the skin. For many topical drugs the active pharmaceutical ingredient is retained in the non-viable outer $\mathrm{SC}$, with only a small fraction reaching the proliferative basal epidermis or viable dermis. While molar $(\mathrm{M})$ concentrations of active drug can be detected in the SC, reports for multiple drugs in human and/or minipig skin have detected substantially lower (nM to $\mu \mathrm{M}$ ) concentrations at skin locations more relevant for carcinogenicity risk, such as the epidermal stratum basale.

tum basale) compared to the SC, and similarly low concentrations of drug in the dermis as reported with other compounds by OFM (Hollingshead et al., 2021). This skin epidermal concentration gradient in tissue collected by the LCM method is similar to that reported for the topical calcineurin inhibitor, pimecrolimus, by quantification of radiolabeled compound (Hultsch et al., 2005).

Concentrations of drug in the proliferative epidermal stem cells of the stratum basale and dermis are likely most relevant for understanding potential dose-related carcinogenic risk. As described above, and illustrated in Fig. 1, the concentrations of many topical drugs in the dermis and basal epidermis are present in the nanomolar and low micromolar range. These are concentrations that are often achieved in plasma in the context of an oral toxicity study. Therefore, it is possible that concentrations of drug achieved in the skin by oral toxicity studies in rodents for compounds with suitable volumes of distribution may be sufficiently high to cover the anticipated exposure in humans by topical application (Rodgers et al., 2005; Rodgers and Rowland, 2006). In these cases, an appropriately conducted oral carcinogenicity study may adequately support a dermal carcinogenicity risk evaluation without need to conduct an additional 2-year dermal carcinogenicity study consistent with guidance from ICH S1A.

\section{Genetic toxicity profile}

All small molecule drug development candidates will be evaluated in a battery of in vitro and in vivo assays, which are intended for hazard identification of a compounds ability to induce gene mutations, structural and/ or numerical chromosomal aberration (ICH S2(R1)). The test battery usually consists of the bacterial Ames assay to identify gene mutagens, a mammalian test for clastogenicity and aneugenicity and an in vivo test for chromosomal aberrations in the blood or bone marrow of exposed animals.

Compounds that induce gene mutations in the bacterial Ames assay have a high likelihood of being carcinogens and are usually removed from drug development consideration unless the indication justifies. Similarly, clastogens without mechanistic information indicating low risk for genotoxicity are rarely progressed to clinical development. A special case is usually made for aneugens that are not expected to interact with DNA directly, but impact structures involved in the accurate distribution of chromosomes during mitosis (Aardema et al., 1998). Aneuploidy inducing agents do generally not induce gene mutations but the loss or gain of whole chromosomes via threshold mechanisms (Elhajouji et al., 2011, 1997, 1995). The best described mechanism for aneuploidy induction is probably tubulin binding, which causes mitotic spindle abnormalities leading to chromosome segregation errors and aneuploidy induction (Lynch et al., 2019). In addition, kinase inhibitors in drug development are frequently shown to be in vitro aneugens via off-target inhibition of kinases involved in the regulation of mitotic processes like aurora kinase B (Sasaki et al., 2020). Both, tubulin binding and aurora $\mathrm{B}$ inhibition are mechanisms that are highly toxic to dividing cells and induce very frequently toxicities in the gastrointestinal tract and in the bone marrow (Bates and Eastman, 2017; Bavetsias and Linardopoulos, 2015; Falchook et al., 2015).

Systemically applied drugs with aneuploidy findings are usually risk managed using safety margin arguments comparing the no effect level concentration in the in vivo micronucleus studies with the systemic exposure in humans.

For topically applied compounds, the determination of safety margins is somewhat more complicated. Since aneuploidy can only be detected in dividing cells, aneuploidy induction in the skin focuses mainly on the divid- 
Topical carcinogenicity challenges

ing keratinocytes of the stratum basale. As indicated above, only a small percentage of a drug applied topically will likely reach the stratum basale and the accurate determination of compound concentration in this compartment has been rather difficult. In addition, no validated in vivo aneugenicity assessment method for human or minipig skin exists, adding to the difficulty for the evaluation of drug candidates. For this reason, we have assessed the suitability of the reconstructed skin micronucleus assay in the EpiDerm ${ }^{\mathrm{TM}}$ model for the in vitro assessment of topically applied aneugenic compounds (Schuler et al., 2021). In addition, we looked for mechanistic key event biomarkers like increases in the mitotic index and polyploidy induction that could be characteristic for aneugenic effects in the dividing keratinocytes. While the EpiDerm $^{\mathrm{TM}}$ system was very sensitive for micronucleus and biomarker induction, no significant difference in the dose -response was observed whether the dose was applied topically or has direct access to the cells of interest when dosed via the media. Based on these results, it became clear that a significant limitation of the in vitro EpiDerm $^{\mathrm{TM}}$ appears to be the absence of barrier integrity to limit compound absorption like what would be expected in human and minipig skin, especially when acetone is used as a solvent. This is further supported by an in vivo experiment in the same publication that applied significantly higher doses of aneugens, when compared to the in vitro EpiDerm ${ }^{\mathrm{TM}}$ experiments, topically to the skin of minipigs and did not show evidence of the induction of key event biomarkers (Schuler et al., 2021). These disparate results highlight the known importance of the barrier function of skin that likely does not allow adequate concentrations of even potent aneugens to reach the stratum basale. Additionally, colchicine has been tested in human clinical trials using up to $1 \%$ or $10 \mathrm{mg} / \mathrm{mL}$, concentrations greatly exceeding those resulting in positive $\mathrm{MN}$ response in the EpiDerm ${ }^{\mathrm{TM}}$ model, for actinic keratoses and recalcitrant psoriasis for up to 12 weeks with no changes in the skin or systemic toxicity. (Akar et al., 2001; Faghihi et al., 2016; Kaidbey et al., 1975). The overall weight of evidence indicates that aneugens, even at high doses, are unlikely to be aneugenic in human skin presumably due to its barrier function preventing sufficient exposure to the stratum basale.

Lastly, while aneuploidy is regarded as a hallmark of cancer, its role in the carcinogenic process is complex. A recent International Workshop on Genotoxicity Testing reviewed the role of aneuploidy in cancer biology; cancer risk associated with constitutive aneuploidy; rodent carcinogenesis with known chemical aneugens; and chemotherapy-related malignant neoplasms (Tweats et al., 2019). The expert working group concluded that the aneugens reviewed that induce cancer in humans and animals all possess other carcinogenic properties, such as mutagenicity, clastogenicity, cytotoxicity, organ toxicities, hormonal, and epigenetic changes which likely account for, or interact with aneuploidy, to cause carcinogenesis. Tubulin-disrupting aneugens that do not possess other properties linked to carcinogenesis, were not carcinogenic in rodents (EMEA, 2001; IARC, 1981a, 1981b; Longstaff and von Krogh, 2001; Takeda, 2017). In fact, colchicine significantly reduces the incidence cancer in a 12-year cohort study in male gout patients (Kuo et al., 2015).

Based on the overall weight of evidence, we conclude that for aneugens that do not show cytotoxicity in the skin, and do not possess other confounding properties like clastogenicity or mutagenicity, there should be no need for topical carcinogenicity studies.

\section{REVIEW OF MARKETED TOPICAL DRUG NONCLINICAL CARCINOGENICITY PACKAGES}

To examine the nonclinical strategies used to characterize carcinogenicity risk for FDA and PMDA approved topical drugs we reviewed available summary information from the Drugs@FDA database (www.accessdata. fda.gov/scripts/cder/daf/) and for PMDA Drug Review reports (www.pmda.go.jp/english/review-services/ reviews/approved-information/drugs/0001.html) available in English. We focused on topical drugs that were granted marketing authorization within the past 25 years by the FDA intended for longer than intermittent clinical use to both review the scope of nonclinical carcinogenicity packages, but also to determine if trends in strategy for assessing such risks have evolved during that time. We also reviewed the summary documents for two (sirolimus and delgocitinib) recently approved topical drugs in Japan. It should be noted that summary review documents contain variable amounts of detail for study types, results, and overall interpretations. Therefore, this assessment is not intended to be a review of all topical drugs approved during this timeframe, and data summarized in Table 1 should be interpreted with the understanding that information available to review in these documents are not comprehensive and can contain inconsistencies. Nevertheless, a holistic analysis of carcinogenicity packages across these approved products highlights trends and provide useful case studies for overall weight of evidence considerations of scenarios for the value of topical carcinogenicity studies.

The carcinogenicity packages for 23 topical drug products approved between 1997 and 2020 are listed in 
B.D. Hollingshead et al.

Table 1. Carcinogenicity packages for approved topical drugs.

\begin{tabular}{|c|c|c|c|c|c|c|c|}
\hline \multirow{2}{*}{ Drug } & \multirow{2}{*}{\begin{tabular}{|c|} 
Topical \\
Approval \\
\end{tabular}} & \multicolumn{2}{|c|}{ Oral Carcinogenicity } & \multicolumn{2}{|c|}{ Topical Carcinogenicity } & \multicolumn{2}{|c|}{ Photocarcinogenicity } \\
\hline & & Type & $+/-$ & Type & $+/-$ & Type & $+/-$ \\
\hline \multirow{2}{*}{ Minoxidil } & \multirow{2}{*}{1997} & 2-year rat & - & 2-year rat & $\begin{array}{c}+ \\
\text { (adrenal, preputial gland) }\end{array}$ & \multirow{2}{*}{ 1-year hairless mouse } & \multirow{2}{*}{ - } \\
\hline & & 2-year mouse & $\begin{array}{c}+ \\
\text { (lymphoma) }\end{array}$ & 2-year mouse & - & & \\
\hline \multirow{2}{*}{ Diclofenac } & \multirow{2}{*}{2000} & 2 -year rat & - & \multirow{2}{*}{ 2-year mouse } & \multirow{2}{*}{ - } & \multirow{2}{*}{ 1-year hairless mouse } & \multirow{2}{*}{+} \\
\hline & & 2-year mouse & - & & & & \\
\hline \multirow{2}{*}{ Tacrolimus } & \multirow{2}{*}{2000} & 2-year rat & - & \multirow{2}{*}{ 2-year mouse } & \multirow{2}{*}{$\begin{array}{c}+ \\
\text { (lymphoma) }\end{array}$} & \multirow{2}{*}{ 1-year hairless mouse } & \multirow{2}{*}{+} \\
\hline & & 80-week mouse & - & & & & \\
\hline \multirow{2}{*}{ Pimecrolimus } & \multirow{2}{*}{2001} & Two 2-year rat & $\begin{array}{c}+ \\
\text { (thymoma) } \\
\end{array}$ & 2-year rat & $\begin{array}{c}+ \\
\text { (thyroid) }\end{array}$ & \multirow{2}{*}{ 1-year hairless mouse } & \multirow{2}{*}{$\stackrel{+}{+}$} \\
\hline & & 2-year mouse & $\begin{array}{c}+ \\
\text { (lymphoma) }\end{array}$ & 2-year mouse & - & & \\
\hline Azelaic acid & 2002 & None & & $\begin{array}{l}\text { 6-month Tg.AC and } \\
\text { 2-year dermal mouse } \\
\text { (post marketing) }\end{array}$ & - & None & \\
\hline Dapsone & 2005 & 2-year rat & - & 6-month Tg.AC mouse & - & 1-year hairless mouse & - \\
\hline Desonide & 2006 & None & & $\begin{array}{c}\text { 6-month Tg.AC mouse } \\
\text { (post marketing) }\end{array}$ & $\begin{array}{c}+ \\
(\text { skin) } \\
+ \\
\text { in vitro mouse } \\
\text { lymphoma assay } \\
\text { (potential mutagen) }\end{array}$ & None & \\
\hline Imiquimod & 2010 & 2-year rat & - & 2-year mouse & $\begin{array}{c}+ \\
\text { (liver) }\end{array}$ & 1-year hairless mouse & - \\
\hline Adapalene & 2010 & 2 -year rat & $\begin{array}{c}+ \\
\text { (adrenal, pancreas, thyroid) }\end{array}$ & 2-year mouse & - & None & \\
\hline Ivermectin & 2012 & None & & None & & None & \\
\hline Igenol mebutate & 2012 & None & & None & & None & \\
\hline Luliconazole & 2013 & None & & None & & None & \\
\hline \multirow[b]{2}{*}{ Brimonidine } & \multirow[b]{2}{*}{2013} & 2-year rat & - & \multirow[b]{2}{*}{ 2-year rat } & \multirow[b]{2}{*}{ - } & 1-year hairless mouse & \\
\hline & & 2-year mouse & - & & & $\begin{array}{l}\text { (recommended prior } \\
\text { to ICHM3 revision) }\end{array}$ & - \\
\hline Ivermectin & 2014 & 2 -year rat & $\begin{array}{c}+ \\
\text { (liver) }\end{array}$ & 2-year mouse & - & None & \\
\hline efinaconazole & 2014 & $\begin{array}{c}\text { None } \\
\text { (waiver) }\end{array}$ & & 2-year mouse & - & None & \\
\hline Tavaborole & 2014 & 2-year rat & - & 2-year mouse & - & None & \\
\hline Crisaborole & 2016 & 2-year rat & $\begin{array}{c}+ \\
+ \\
\text { (uterus) }\end{array}$ & 2-year mouse & - & None & \\
\hline $\begin{array}{l}\text { Oxymetazoline } \\
\text { hydrochloride }\end{array}$ & 2017 & $\begin{array}{l}\text { 6-month transgenic mouse } \\
\text { (waiver for 2-year, } \\
\text { would not be tolerated } \\
\text { based on 6-month tox) }\end{array}$ & - & None & & None & \\
\hline Ozenoxacin & 2017 & None & & None & & None & \\
\hline Trifarotene & 2019 & 2-year rat & - & 2-year mouse & - & None & \\
\hline Sirolimus & 2018 & 2-year mouse & $\begin{array}{c}+ \\
\text { (leukemia, liver) }\end{array}$ & Mid-term mouse skin & - & None & \\
\hline (Japan) & 2018 & 2-year rat & $\begin{array}{c}+ \\
\text { (testes) }\end{array}$ & carcinogenicity & - & None & \\
\hline Minocycline & 2019 & 2-year rat & $\begin{array}{c}+ \\
\text { (thyroid) }\end{array}$ & $\begin{array}{l}\text { None } \\
\text { (Waiver) }\end{array}$ & & None & \\
\hline & & 2-year mouse & - & & & & \\
\hline $\begin{array}{l}\text { Delgocitinib } \\
\text { (Japan) }\end{array}$ & 2020 & 2-year rat & $\begin{array}{c}+ \\
\text { (thymus, testes, } \\
\text { pancreas, subcutis) }\end{array}$ & 2-year mouse & - & None & \\
\hline Clascoterone & 2020 & None & & 2-year rat & - & None & \\
\hline
\end{tabular}

- = no test article-related tumor findings

SBA References: (Pharmaceuticals and Medical Devices Agency, 2014; Pharmaceuticals and Medical Devices Agency, 2018; Pharmaceuticals and Medical Devices Agency, 2019; US Food and Drug Administration, 1997; US Food and Drug Administration, 2000a; US Food and Drug Administration, 2000b; US Food and Drug Administration, 2001; US Food and Drug Administration, 2002; US Food and Drug Administration, 2005; US Food and Drug Administration, 2006; US Food and Drug Administration, 2010a; US Food and Drug Administration, 2010b; US Food and Drug Administration, 2012a; US Food and Drug Administration, 2012b; US Food and Drug Administration, 2013a; US Food and Drug Administration, 2013b; US Food and Drug Administration, 2014a; US Food and Drug Administration, 2014b; US Food and Drug Administration, 2014c; US Food and Drug Administration, 2016; US Food and Drug Administration, 2017a; US Food and Drug Administration, 2017b; US Food and Drug Administration, 2019a; US Food and Drug Administration, 2019b; US Food and Drug Administration, 2020)

Vol. 47 No. 1 
Topical carcinogenicity challenges

Table 1. The overall breadth of these study packages varies but generally reflects a trend in more recent approvals for lower number of carcinogenicity studies. The absence of photocarcinogenicity studies after $\sim 2013$ is expected since reduction of this study type was recommended in the 2009 ICH multidisciplinary guidance revision (ICHM3 (R2), Note 6). The topical products listed in Table 1 that were approved between 1997 and 2001 (minoxidil, diclofenac, tacrolimus, and pimecrolimus) completed carcinogenicity study packages inclusive of oral, topical, and photocarcinogenicity studies. These contained 2-year oral carcinogenicity studies in rats and mice, a 2-year topical carcinogenicity study in mice (rat and mouse for minoxidil), and a mouse photocarcinogenicity study for a total of 4-5 carcinogenicity studies to support each products marketing application. The large number of studies conducted for these drugs likely are reflective of a combination of two elements: 1) design and execution of studies prior to ICH S1 guidance implementation and/ or 2) prior or concurrent development of the active pharmaceutical compound for systemic treatments. The total number of carcinogenicity studies summarized to support a topical drug approval after 2001 typically range from 0 to 3 . The rationale for not conducting carcinogenicity studies for some products was based on limited duration of use (e.g., lice (ivermectin), actinic keratosis (ingenol mebutate), or infection (ozenoxacin)) or low systemic bioavailability combined with absence of preneoplastic effects in chronic minipig toxicity studies (e.g., antifungal (luliconazole)) (US Food and Drug Administration, 2012a, 2012b, 2013a, 2017b). Ivermectin, for example, did not submit any carcinogenicity studies to support its use for the treatment of lice in 2012 based on its limited duration of use clinically, but its 2014 approval for use in treating Rosacea included submission of carcinogenicity data from 2-year oral rat and 2-year topical mouse studies (US Food and Drug Administration, 2014c).

It is noteworthy that for all compounds summarized in Table 1, there are no examples of test article-related skin tumors occurring at the site of drug application in 2 -year topical rodent carcinogenicity studies. It is possible that this selection of drugs is skewed towards favorable profiles since it only represents approved drug products. However, it does also highlight the possibility that the rodent may not be sensitive for detecting skin carcinogenicity risk, or that skin is not a sensitive target in these studies, particularly since other types of tumors (e.g., liver, adrenal, thyroid, etc.) were observed in some topical studies in the absence of skin tumors. The only example that detected positive evidence for test article-related skin tumors was from a Tg. AC transgenic mouse model for desonide, but the relevance of that finding to human was considered uncertain and results confounded by the possible mutagenic activity identified in vitro (US Food and Drug Administration, 2006). Additionally, while the Tg.AC mouse carcinogenicity assay has been utilized as an alternative model for some products the relevance of this model to human risk is unclear and it is no longer used in the United States (Jacobs and Brown, 2015).

Interestingly, minoxidil, tacrolimus, and imiquimod each conducted oral and topical rodent carcinogenicity studies, but only identified drug-related tumors in the topical studies at locations outside of the application site. While some of these observations could be related to strain sensitivities to tumors (Friedrich and Olejniczak, 2011) it is also likely that tumors were observed in topical studies but not oral studies for these drugs due to systemic exposure differences. Tacrolimus, for example, noted lymphoma in the 2-year topical mouse, but not in the 2 -year oral rat or 80-week mouse carcinogenicity studies. The observation of lymphoma in the topical mouse study, however, occurred at higher plasma levels than achieved in either of the oral studies and at large exposures relative to the clinically relevant human levels. In fact, the plasma exposures at the no observed effect level for tumors in the topical and mouse oral carcinogenicity studies were almost identical (US Food and Drug Administration, 2000a). Similarly, the finding of imiquimod-related liver tumors in the 2-year mouse topical carcinogenicity study occurred at higher plasma exposures than those achieved in the 2-year oral rat study (US Food and Drug Administration, 2010b). These cases serve as cautionary examples of the point introduced earlier regarding the high permeability of drugs when applied to in rodent skin compared to human and minipig.

Conversely, increases in delgocitinib-related tumors (thymus, testes, pancreas, subcutis) were identified in the 2 -year oral rat carcinogenicity study, but not the 2-year topical mouse study. While species differences could account for some of these observations all delgocitinibrelated tumor findings were observed at doses achieving higher plasma exposures than those achieved in the dermal mouse study. Delgocitinib is an interesting case study since higher incidence of epidermal hyperplasia was observed in the chronic dermal minipig toxicity study in delgocitinib-containing formulations (Common Tabulated Document, Section 2.6), but the 2-year dermal mouse carcinogenicity study was negative for increased incidence of compound-related skin tumors (Pharmaceuticals and Medical Devices Agency, 2019). This minipig microscopic finding could be interpreted as a signal for neoplastic risk, however, it is noteworthy that incidence of epider- 
B.D. Hollingshead et al.

mal hyperplasia was similar to control animals following completion of a 1-month recovery phase. This recovery data supports that the epidermal hyperplasia at the end of the dosing phase was not in indicator of neoplastic risk, but rather an normal physiologic response to skin irritation (Toussaint et al., 1999), and is a reminder of the importance of incorporating expert pathology interpretation in the overall risk assessment for a product.

Taken together, a review of 23 drugs approved for topical application show a declining trend toward conduct of topical rodent carcinogenicity studies, with test article associated tumors related to systemic exposures not local application site effects. The absence of identified skin risks in rodent carcinogenicity studies highlights the value of evaluating skin effects in more human relevant minipig chronic toxicity studies to determine if any human risks are present.

\section{CONCLUSION}

While a complete nonclinical safety package for topical drugs is generally similar to other routes of administration there are considerations that could necessitate the conduct of additional studies or elimination of others. We have summarized above some of the main considerations that should be evaluated as part of the overall assessment of skin carcinogenicity risk of a topical product and suggest a decision process for determining if a 2-year topical rodent carcinogenicity study would add to the overall carcinogenicity risk assessment. The schematic in Fig. 2 is focused on topical drugs that are intended for longer than intermittent clinical use and have some systemic exposure liabilities since as mentioned earlier there are examples of topical drugs that did not require any studies based on these criteria. Additionally, this decision process may not be applicable if inactive ingredients in a novel drug product are unprecedented.

Fundamentally, the decision process outlined in Fig. 2 considers that if skin exposures are below levels of concern and within ranges achieved by oral studies in rodents then there is limited utility in conducting additional topical carcinogenicity studies in rodents. It also incorporates in the decision process an assessment of skin findings from the minipig chronic toxicity study and overall genetic toxicity risk profile to determine if any signal for concern is present. While there are without doubt circumstances that will necessitate the need to conduct rodent carcinogenicity studies based on absence of comprehensive supportive information, these studies should not be viewed as obligate when sufficient supportive information already exists for the overall weight of evidence risk

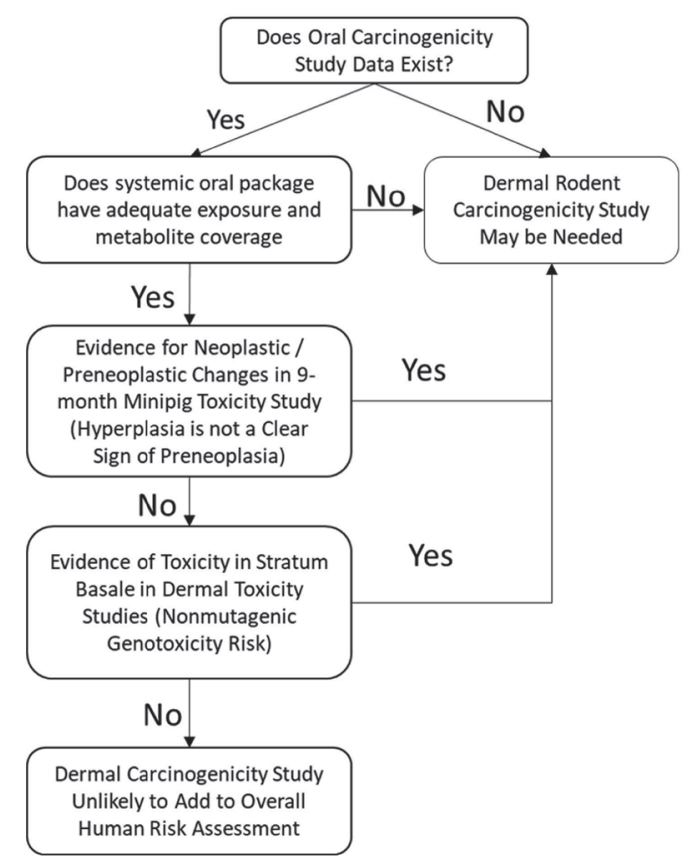

Fig. 2. A set of decision criteria are outlined to determine if a topical drug in clinical development should consider conducting a topical rodent carcinogenicity study or if it is unlikely to add to the overall human risk assessment. The supportive information that should be considered should include, but not necessarily limited to, existing systemic toxicity and carcinogenicity study data, anticipated exposures in skin and plasma, relative risk based on skin evaluation in a chronic minipig toxicity study, and genetic toxicity risk profile.

assessment. The overarching principals guiding this dermal carcinogenesis study decision process do have precedent based on the summary approval packages for minocycline (US Food and Drug Administration, 2019b) and luliconazole (US Food and Drug Administration, 2013a), both of which received dermal carcinogenicity study waivers due to absence of neoplastic concern from other studies, with particular focus on the chronic minipig results. In summary, conducting 2-year dermal carcinogenicity studies in rodents only when scientifically justified will also aid to achieve the 3 Rs principles for reduction of unnecessary animal testing without compromising safety and effectiveness for topical drug products.

Conflict of interest---- The authors of this manuscript were employed by Pfizer, Inc. during the conduct of this work and are shareholders of Pfizer Inc. stock. 
Topical carcinogenicity challenges

\section{Abbreviations}

International Council for Harmonisation (ICH), Food and Drug Administration (FDA), Pharmaceuticals and Medical Devices Agency (PMDA), stratum corneum (SC), open flow microperfusion (OFM), laser capture microdissection (LCM)

\section{REFERENCES}

Aardema, M.J., Albertini, S., Arni, P., Henderson, L.M., KirschVolders, M., Mackay, J.M., Sarrif, A.M., Stringer, D.A. and Taalman, R.D. (1998): Aneuploidy: a report of an ECETOC task force. Mutat. Res., 410, 3-79.

Akar, A., Bülent Taştan, H., Erbil, H., Arca, E., Kurumlu, Z. and Gür, A.R. (2001): Efficacy and safety assessment of $0.5 \%$ and $1 \%$ colchicine cream in the treatment of actinic keratoses. J. Dermatolog. Treat., 12, 199-203.

Bartek, M.J., LaBudde, J.A. and Maibach, H.I. (1972): Skin permeability in vivo: comparison in rat, rabbit, pig and man. J. Invest. Dermatol., 58, 114-123.

Bates, D. and Eastman, A. (2017): Microtubule destabilising agents: far more than just antimitotic anticancer drugs. Br. J. Clin. Pharmacol., 83, 255-268.

Bavetsias, V. and Linardopoulos, S. (2015): Aurora Kinase Inhibitors: Current Status and Outlook. Front. Oncol., 5, 278.

Bodenlenz, M., Augustin, T., Birngruber, T., Tiffner, K.I., Boulgaropoulos, B., Schwingenschuh, S., Raney, S.G., Rantou, E. and Sinner, F. (2020): Variability of Skin Pharmacokinetic Data: Insights from a Topical Bioequivalence Study Using Dermal Open Flow Microperfusion. Pharm. Res., 37, 204.

Bodenlenz, M., Tiffner, K.I., Raml, R., Augustin, T., Dragatin, C., Birngruber, T., Schimek, D., Schwagerle, G., Pieber, T.R., Raney, S.G., Kanfer, I. and Sinner, F. (2017): Open Flow Microperfusion as a Dermal Pharmacokinetic Approach to Evaluate Topical Bioequivalence. Clin. Pharmacokinet., 56, 91-98.

Bonnel, D., Legouffe, R., Eriksson, A.H., Mortensen, R.W., Pamelard, F., Stauber, J. and Nielsen, K.T. (2018): MALDI imaging facilitates new topical drug development process by determining quantitative skin distribution profiles. Anal. Bioanal. Chem., 410, 2815-2828.

Dorne, J.L. and Renwick, A.G. (2005): The refinement of uncertainty/safety factors in risk assessment by the incorporation of data on toxicokinetic variability in humans. Toxicol. Sci., 86, 20-26.

Eirefelt, S., Hummer, J., Basse, L.H., Bertelsen, M., Johansson, F., Birngruber, T., Sinner, F., Larsen, J., Nielsen, S.F. and Lambert, M. (2020): Evaluating Dermal Pharmacokinetics and Pharmacodymanic Effect of Soft Topical PDE4 Inhibitors: Open Flow Microperfusion and Skin Biopsies. Pharm. Res., 37, 243.

Elhajouji, A., Lukamowicz, M., Cammerer, Z. and Kirsch-Volders, M. (2011): Potential thresholds for genotoxic effects by micronucleus scoring. Mutagenesis, 26, 199-204.

Elhajouji, A., Tibaldi, F. and Kirsch-Volders, M. (1997): Indication for thresholds of chromosome non-disjunction versus chromosome lagging induced by spindle inhibitors in vitro in human lymphocytes. Mutagenesis, 12, 133-140.

Elhajouji, A., Van Hummelen, P. and Kirsch-Volders, M. (1995): Indications for a threshold of chemically-induced aneuploidy in vitro in human lymphocytes. Environ. Mol. Mutagen., 26, 292-304.

EMEA. (2001): Mebendazole Summary Report (2) EMEA/
MRL/781/01-FINAL

Faghihi, G., Elahipoor, A., Iraji, F., Behfar, S. and Abtahi-Naeini, B. (2016): Topical Colchicine Gel versus Diclofenac Sodium Gel for the Treatment of Actinic Keratoses: A Randomized, DoubleBlind Study. Adv. Med., 2016, 5918393.

Falchook, G.S., Bastida, C.C. and Kurzrock, R. (2015): Aurora Kinase Inhibitors in Oncology Clinical Trials: Current State of the Progress. Semin. Oncol., 42, 832-848.

Feldman, S.R., Gelfand, J.M., Stein Gold, L. and Jones, S.D. (2007): The role of topical therapy for patients with extensive psoriasis. Cutis, 79 (Suppl 2), 18-31.

Florek, A.G., Wang, C.J. and Armstrong, A.W. (2018): Treatment preferences and treatment satisfaction among psoriasis patients: a systematic review. Arch. Dermatol. Res., 310, 271-319.

Friedrich, A. and Olejniczak, K. (2011): Evaluation of carcinogenicity studies of medicinal products for human use authorised via the European centralised procedure (1995-2009). Regul. Toxicol. Pharmacol., 60, 225-248.

Fujii, M., Yamanouchi, S., Hori, N., Iwanaga, N., Kawaguchi, N. and Matsumoto, M. (1997): Evaluation of Yucatan micropig skin for use as an in vitro model for skin permeation study. Biol. Pharm. Bull., 20, 249-254.

Handler, A.M., Eirefelt, S., Lambert, M., Johansson, F., Hollesen Schefe, L., Østergaard Knudsen, N., Bodenlenz, M., Birngruber, T., Sinner, F., Huss Eriksson, A., Pommergaard Pedersen, G., Janfelt, C. and Troensegaard Nielsen, K. (2021): Characterizing Cutaneous Drug Delivery Using Open-Flow Microperfusion and Mass Spectrometry Imaging. Mol. Pharm., 18, 3063-3072.

Herkenne, C., Alberti, I., Naik, A., Kalia, Y.N., Mathy, F.X., Préat, V. and Guy, R.H. (2008): In vivo methods for the assessment of topical drug bioavailability. Pharm. Res., 25, 87-103.

Hernández, L.G., van Benthem, J. and Johnson, G.E. (2013): A mode-of-action approach for the identification of genotoxic carcinogens. PLoS One, 8, e64532.

Hollingshead, B.D., Tomlinson, L., Finley, J., Doshna, C., Ritenour, C., Barricklow, J., Oppenheimer, S.R., O’Neil, S.P., Moore, J.L., Patterson, N.H., Nicholson, S.P., Norris, J.L., Caprioli, R.M., Beaumont, K., King-Ahmad, A.J., Vispute, S., Cook, J.C., Radi, Z. and Schuler, M. (2021): An orthogonal methods assessment of topical drug concentrations in skin and the impact for risk assessment in the viable epidermis. Regul. Toxicol. Pharmacol., 123, 104934

Hultsch, T., Kapp, A. and Spergel, J. (2005): Immunomodulation and safety of topical calcineurin inhibitors for the treatment of atopic dermatitis. Dermatology, 211, 174-187.

IARC. (1981a): Vinblastine sulphate. IARC Monogr. Eval. Carcinog. Risk Chem. Hum., 26, 349-363.

IARC. (1981b): Vincristine sulphate. IARC Monogr. Eval. Carcinog. Risk Chem. Hum., 26, 365-384.

Jacobs, A.C. and Brown, P.C. (2015): Regulatory Forum Opinion Piece*: Transgenic/Alternative Carcinogenicity Assays: A Retrospective Review of Studies Submitted to CDER/FDA $1997-$ 2014. Toxicol. Pathol., 43, 605-610.

Kaidbey, K.H., Petrozzi, J.W. and Kligman, A.M. (1975): Topical colchicine therapy for recalcitrant psoriasis. Arch. Dermatol., 111, 33-36.

Koster, M.I. (2009): Making an epidermis. Ann. N. Y. Acad. Sci., 1170, 7-10.

Kuo, M.C., Chang, S.J. and Hsieh, M.C. (2015): Colchicine Significantly Reduces Incident Cancer in Gout Male Patients: A 12-Year Cohort Study. Medicine (Baltimore), 94, e1570.

Lehr, K.-H. (2011): Toxicokinetics and Safety Ratios. In: Drug 


\section{B.D. Hollingshead et al.}

Discovery and Evaluation: Methods in Clinical Pharmacology (Vogel, H.G. et al., Eds.), pp.245-249, Springer Berlin Heidelberg, Berlin, Heidelberg.

Longstaff, E. and von Krogh, G. (2001): Condyloma eradication: self-therapy with $0.15-0.5 \%$ podophyllotoxin versus $20-25 \%$ podophyllin preparations--an integrated safety assessment. Regul. Toxicol. Pharmacol., 33, 117-137.

Lynch, A.M., Eastmond, D., Elhajouji, A., Froetschl, R., KirschVolders, M., Marchetti, F., Masumura, K., Pacchierotti, F., Schuler, M. and Tweats, D. (2019): Targets and mechanisms of chemically induced aneuploidy. Part 1 of the report of the 2017 IWGT workgroup on assessing the risk of aneugens for carcinogenesis and hereditary diseases. Mutat. Res. Genet. Toxicol. Environ. Mutagen., 847, 403025.

Monro, A. (1993): How useful are chronic (life-span) toxicology studies in rodents in identifying pharmaceuticals that pose a carcinogenic risk to humans? Adverse Drug React. Toxicol. Rev., 12, 5-34.

Mortensen, J.T., Brinck, P. and Lichtenberg, J. (1998): The minipig in dermal toxicology. A literature review. Scan. J. Lab. Anim. Sci., 25 (Suppl 1), 77-83.

OECD. (2009): Test No. 437: Bovine Corneal Opacity and Permeability Test Method for Identifying Ocular Corrosives and Severe Irritants.

OECD. (2018a): Test No. 442D: In Vitro Skin Sensitisation.

OECD. (2018b): Test No. 442E: In Vitro Skin Sensitisation.

OECD. (2020): Test No. 442C: In Vitro Skin Sensitisation.

Olaharski, A.J., Gonzaludo, N., Bitter, H., Goldstein, D., Kirchner, S., Uppal, H. and Kolaja, K. (2009): Identification of a kinase profile that predicts chromosome damage induced by small molecule kinase inhibitors. PLOS Comput. Biol., 5, e1000446.

Pharmaceuticals and Medical Devices Agency. (2014): Rapalimus ${ }^{\mathrm{TM}}$ (sirolimus tablets) Report on the Deliberation Results. Pharmaceutical Evaluation Division.

Pharmaceuticals and Medical Devices Agency. (2018): Rapalimus ${ }^{\mathrm{TM}}$ (sirolimus gel) Report on the Deliberation Results. Pharmaceutical Evaluation Division.

Pharmaceuticals and Medical Devices Agency. (2019): Corectim ${ }^{\mathrm{TM}}$ (delgocitinib ointment) Report on the Deliberation Results. Pharmaceutical Evaluation Division.

Rodgers, T., Leahy, D. and Rowland, M. (2005): Physiologically based pharmacokinetic modeling 1: predicting the tissue distribution of moderate-to-strong bases. J. Pharm. Sci., 94, 1259-1276.

Rodgers, T. and Rowland, M. (2006): Physiologically based pharmacokinetic modelling 2: predicting the tissue distribution of acids, very weak bases, neutrals and zwitterions. J. Pharm. Sci., 95, 1238-1257.

Sasaki, J.C., Allemang, A., Bryce, S.M., Custer, L., Dearfield, K.L., Dietz, Y., Elhajouji, A., Escobar, P.A., Fornace, A.J. Jr., Froetschl, R., Galloway, S., Hemmann, U., Hendriks, G., Li, H.H., Luijten, M., Ouedraogo, G., Peel, L., Pfuhler, S., Roberts, D.J., Thybaud, V., van Benthem, J., Yauk, C.L. and Schuler, M. (2020): Application of the adverse outcome pathway framework to genotoxic modes of action. Environ. Mol. Mutagen., 61, 114-134.

Schuler, M., Tomlinson, L., Homiski, M., Cheung, J., Zhan, Y., Coffing, S., Engel, M., Rubitski, E., Seitis, G., Hales, K., Robertson, A., Vispute, S., Cook, J., Radi, Z. and Hollingshead, B. (2021): Experiments in the EpiDerm 3D Skin In Vitro Model and Minipigs In Vivo Indicate Comparatively Lower In Vivo Skin Sensitivity of Topically Applied Aneugenic Compounds. Toxicol. Sci., 180, 103-121.

Shen, J., Swift, B., Mamelok, R., Pine, S., Sinclair, J. and Attar, M.
(2019): Design and Conduct Considerations for First-in-Human Trials. Clin. Transl. Sci., 12, 6-19.

Sistare, F.D., Morton, D., Alden, C., Christensen, J., Keller, D., Jonghe, S.D., Storer, R.D., Reddy, M.V., Kraynak, A., Trela, B., Bienvenu, J.G., Bjurström, S., Bosmans, V., Brewster, D., Colman, K., Dominick, M., Evans, J., Hailey, J.R., Kinter, L., Liu, M., Mahrt, C., Marien, D., Myer, J., Perry, R., Potenta, D., Roth, A., Sherratt, P., Singer, T., Slim, R., Soper, K., FranssonSteen, R., Stoltz, J., Turner, O., Turnquist, S., van Heerden, M., Woicke, J. and DeGeorge, J.J. (2011): An analysis of pharmaceutical experience with decades of rat carcinogenicity testing: support for a proposal to modify current regulatory guidelines. Toxicol. Pathol., 39, 716-744.

Stricker-Krongrad, A., Shoemake, C.R., Liu, J., Brocksmith, D. and Bouchard, G. (2017): The importance of minipigs in dermal safety assessment: an overview. Cutan. Ocul. Toxicol., 36, 105-113.

Surber, C., Wilhelm, K.P., Bermann, D. and Maibach, H.I. (1993): In vivo skin penetration of acitretin in volunteers using three sampling techniques. Pharm. Res., 10, 1291-1294.

Takeda. (2017): Colcrys Prescription information.

Tieppo Francio, V., Davani, S., Towery, C. and Brown, T.L. (2017): Oral Versus Topical Diclofenac Sodium in the Treatment of Osteoarthritis. J. Pain Palliat. Care Pharmacother., 31, 113-120.

Toussaint, S., Salcedo, E. and Kamino, H. (1999): Benign epidermal proliferations. Adv. Dermatol., 14, 307-357.

Trottet, L.M. (2017): Assessing Drug Concentration in Skin: Direct and Indirect Methods. In: Dermal Drug Selection and Development, pp.49-67, Springer, Cham.

Tweats, D., Eastmond, D.A., Lynch, A.M., Elhajouji, A., Froetschl, R., Kirsch-Volders, M., Marchetti, F., Masumura, K., Pacchierotti, F. and Schuler, M. (2019): Role of aneuploidy in the carcinogenic process: Part 3 of the report of the 2017 IWGT workgroup on assessing the risk of aneugens for carcinogenesis and hereditary diseases. Mutat. Res. Genet. Toxicol. Environ. Mutagen., 847, 403032

US Food and Drug Administration. (1997): Rogaine ${ }^{\mathrm{TM}}$ (minoxidil topical solution) Summary Basis of Approval. Center for Drug Evaluation and Research.

US Food and Drug Administration. (2000a): Protopic ${ }^{\mathrm{TM}}$ (tacrolimus ointment) Summary Basis of Approval. Center for Drug Evaluation and Research.

US Food and Drug Administration. (2000b): Solaraze ${ }^{\mathrm{TM}}$ (diclofenac sodium gel) Summary Basis of Approval. Center for Drug Evaluation and Research.

US Food and Drug Administration. (2001): Elidel ${ }^{\mathrm{TM}}$ (pimecrolimus cream) Summary Basis of Approval. Center for Drug Evaluation and Research.

US Food and Drug Administration. (2002): Finacea ${ }^{\mathrm{TM}}$ (azelaic acid gel) Summary Basis of Approval. Center for Drug Evaluation and Research.

US Food and Drug Administration. (2005): Aczone ${ }^{\mathrm{TM}}$ (dapsone gel) Summary Basis of Approval. Center for Drug Evaluation and Research.

US Food and Drug Administration. (2006): Desonate ${ }^{\mathrm{TM}}$ (desonide gel) Summary Basis of Approval. Center for Drug Evaluation and Research.

US Food and Drug Administration. (2010a): Differin ${ }^{\mathrm{TM}}$ (adapalene lotion) Summary Basis of Approval. Center for Drug Evaluation and Research.

US Food and Drug Administration. (2010b): Zyclara ${ }^{\mathrm{TM}}$ (imiquimod cream) Summary Basis of Approval. Center for Drug Evaluation and Research. 
Topical carcinogenicity challenges

US Food and Drug Administration. (2012a): Picato ${ }^{\mathrm{TM}}$ (ingenol mebutate gel) Summary Basis of Approval. Center for Drug Evaluation and Research.

US Food and Drug Administration. (2012b): Sklice ${ }^{\mathrm{TM}}$ (ivermecin lotion) Summary Basis of Approval. Center for Drug Evaluation and Research.

US Food and Drug Administration. (2013a): Luzu ${ }^{\mathrm{TM}}$ (luliconazole cream) Summary Basis of Approval. Center for Drug Evaluation and Research.

US Food and Drug Administration. (2013b): Mirvaso ${ }^{\mathrm{TM}}$ (brimonidine gel) Summary Basis of Approval. Center for Drug Evaluation and Research.

US Food and Drug Administration. (2014a): Jublia ${ }^{\mathrm{TM}}$ (efinaconazole solution) Summary Basis of Approval. Center for Drug Evaluation and Research.

US Food and Drug Administration. (2014b): Kerydin ${ }^{\mathrm{TM}}$ (tavaborole solution) Summary Basis of Approval. Center for Drug Evaluation and Research.

US Food and Drug Administration. (2014c): Soolantra ${ }^{\mathrm{TM}}$ (ivermectin cream) Summary Basis of Approval. Center for Drug Evaluation and Research.

US Food and Drug Administration. (2016): Eucrisa ${ }^{\mathrm{TM}}$ (crisaborole ointment) Summary Basis of Approval. Center for Drug Evaluation and Research.

US Food and Drug Administration. (2017a): Rhofade ${ }^{\mathrm{TM}}$ (oxymetazoline hydrochloride cream) Summary Basis of Approval. Center for Drug Evaluation and Research.

US Food and Drug Administration. (2017b): Xepi ${ }^{\mathrm{TM}}$ (ozenoxacin cream) Summary Basis of Approval. Center for Drug Evaluation and Research.

US Food and Drug Administration. (2019a): Aklief ${ }^{\mathrm{TM}}$ (trifarotene cream) Summary Basis of Approval. Center for Drug Evaluation and Research.

US Food and Drug Administration. (2019b): Amzeeq ${ }^{\mathrm{TM}}$ (minocycline foam) Summary Basis of Approval. Center for Drug Evaluation and Research.

US Food and Drug Administration. (2020): Winlevi ${ }^{\mathrm{TM}}$ (clascoterone cream) Summary Basis of Approval. Center for Drug Evaluation and Research.

Wei, J.C., Edwards, G.A., Martin, D.J., Huang, H., Crichton, M.L. and Kendall, M.A. (2017): Allometric scaling of skin thickness, elasticity, viscoelasticity to mass for micro-medical device translation: from mice, rats, rabbits, pigs to humans. Sci. Rep., 7, 15885. 